\title{
Apps, educación y patrimonio en México. Análisis de situación y estudio com- parativo con el caso español ${ }^{1}$
}

\author{
Úrsula Luna*, Pedro José Canto Herrera**, Alfredo Zapata González**, Alex Ibáñez-Etxeberria*2
}

(*) Universidad del País Vasco UPV/EHU - Donostia-San Sebastián, España

$\left.{ }^{* *}\right)$ Universidad Autónoma de Yucatán -Mérida, México

\begin{abstract}
RESUMEN
Este trabajo analiza desde un punto de vista educativo la oferta de apps en torno al patrimonio mexicano. Tras realizar una pequeña revisión de la situación del patrimonio en México y de la implementación de tecnologías digitales, especialmente apps, en el mismo, se hace un breve repaso al estado de la cuestión de la evaluación de apps de contenido patrimonial desde el punto de vista educativo. El estudio, de carácter descriptivo, se realiza desde la perspectiva del estudio de programas siguiendo el método OEPE adaptado al caso de las apps. Los resultados del análisis de la muestra final de 45 apps, muestran similitudes con el contexto español en cuanto a una baja cantidad de apps patrimoniales que puedan considerarse educativas, con escasa atención a la accesibilidad y sin adaptación a diferente tipología de público, prevaleciendo los contenidos y sin un diseño didáctico adecuado. Las diferencias principales se centran en los desarrolladores de apps, que en México son las empresas que crean contenidos dirigidos al turismo, frente a las administraciones públicas y museos en España que ofrecen un discurso patrimonial diverso. Sin embargo, en ambos países encontramos una necesidad de desarrollar apps de calidad que favorezcan su uso educativo.
\end{abstract}

Palabras Clave: app, educación patrimonial, patrimonio, aprendizaje, México

\section{Apps, education and heritage in Mexico. Situation analysis and comparative study with the Spanish case}

\begin{abstract}
This work aims at getting to know the offer of Mexican heritage apps from an educational point of view. After carrying out a small review of the situation of the heritage in Mexico and the implementation of digital technologies, especially apps, in it, a brief review is made of the status of the evaluation of heritage content apps from the educational point of view. This descriptive nature' study, is carried out from the perspective of the study of programs following the SHEO method adapted to the case of apps. The results of the analysis of the final sample of 45 apps, show similarities with the Spanish context in terms of a low number of heritage apps that can be considered educational, with little attention to accessibility and without adaptation to different types of audiences, the contents prevailing and without an adequate didactic design. The main differences focus on app developers, which in Mexico are companies that create content aimed at tourism, compared to public administrations and museums in Spain that offer a diverse heritage discourse. However, in both countries we find a need to develop quality apps that favor their educational use.
\end{abstract}

Key Words: app, heritage education, heritage, learning, México

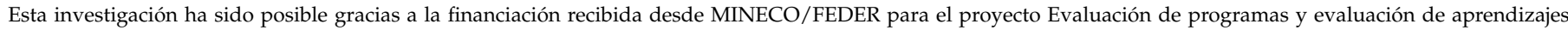

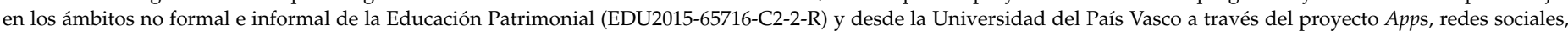

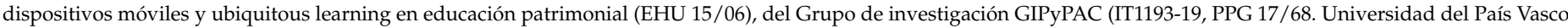

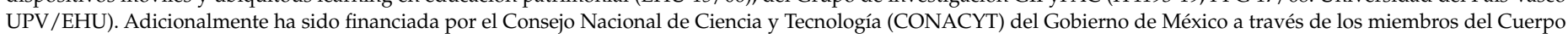
Académico consolidado de Currículo e Instrucción de la Universidad Autónoma de Yucatán.

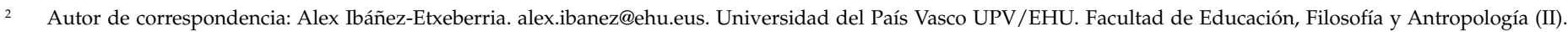
Plaza Oñati, 3. 20018, Donostia-San Sebastián, España.
} 


\section{Introducción}

Este trabajo analiza desde un punto de vista educativo la oferta de apps en torno al patrimonio mexicano. Para ello se realiza en primer lugar una pequeña revisión de la situación del patrimonio en México, así como de la implementación de las tecnologías digitales, especialmente de apps en su comunicación, y posteriormente se hace un breve repaso al estado de la cuestión de la evaluación de apps de contenido patrimonial desde el punto de vista educativo. El estudio se realiza desde la perspectiva del estudio de programas, realizando un análisis de tipo descriptivo siguiendo el método OEPE adaptado al caso de las apps, discutiéndose los resultados con los obtenidos anteriormente en el caso español.

\section{El patrimonio en México}

El patrimonio en México es gestionado mayormente por el Instituto Nacional de Antropología e Historia (INAH) en museos, sitios arqueológicos y monumentos, siendo su objetivo proteger el patrimonio nacional (Miranda, 2016). De acuerdo a UNESCO, el país cuenta con 34 sitios inscritos en la Lista de Patrimonio Mundial, 429 manifestaciones de patrimonio inmaterial, y 8 expresiones culturales en la Lista Representativa del Patrimonio Cultural Inmaterial de la Humanidad (UNESCO, 2017).

En relación con el turismo, México ocupa el sexto puesto mundial en recepción de turistas (Sectur, 2017), siendo el patrimonio tanto cultural como natural, uno de los reclamos principales, y sus museos y zonas arqueológicas, un importante motor de desarrollo. Solo de enero a julio de 2019 se contabilizaron 16 millones y medio de visitantes (Datatur, 2019), desarrollándose al albur de este potencial, interesantes proyectos de turismo cultural patrimonial como el de los Pueblos Mágicos (Fernández, 2016) o la Ruta de los Dioses (Sectur, 2011).

En este contexto, las instituciones mexicanas que se dedican al patrimonio tienen como objetivo y reto transmitir el conocimiento, enfrentándose a la creación de nuevos contenidos y a su vez buscando la manera de hacer accesibles estos contenidos a los ciudadanos (Jiménez-Badillo, 2016), pero, aunque el INAH ha realizado un exhaustivo trabajo de investigación, conservación, restauración y protección del patrimonio, apenas ha desarrollado el ámbito de la socialización de los valores patrimoniales (Gándara, 2016) en el que encuadraríamos el aspecto educativo.

\section{Tecnologías digitales y patrimonio en México}

Según el gobierno mexicano, en 2018 existían más de 71.3 millones de usuarios de Internet, que representaban el 63.9\% de la población mayor de seis años (Gobierno de México, 2018). Complementariamente, un estudio de la Asociación Internet.MX revela que el $89 \%$ de los usuarios utilizan preferentemente para esa conexión el teléfono inteligente o smartphone (AMIPCI, 2018).

En el ámbito de las tecnologías digitales unidas al patrimonio, encontramos en 2015 la creación de la Red de Humanidades Digitales, cuyo objetivo es buscar "la manera de extender el uso de estas herramientas en el campo de la academia y la cultura" (Miranda, 2016:35). Sobre este germen se creó la Red Temática de Tecnologías Digitales para la Difusión del Patrimonio Cultural (Jímenez-Badillo, 2016; RedTDPC, 2016), uno de cuyos logros es la realización anual del Congreso Internacional de Patrimonio Cultural y Nuevas Tecnologías, en una de cuyas primeras ediciones se expusieron tres interesantes conclusiones para el panorama mexicano: Por un lado, se destaca que en relación a la cantidad enorme de patrimonio que alberga el país, no existe una línea de actuación clara en torno a la preservación e investigación del patrimonio y la aplicación de las tecnologías en estos procesos, realizándose acciones dispersas e inconexas. Por otro, que el uso de estas tecnologías en la difusión del patrimonio es casi nulo, limitando las infinitas posibilidades que ofrecen estas herramientas. Y, por último, que la mayoría de los trabajos o proyectos surgen de empresas privadas, quedando las instituciones que salvaguardan el patrimonio ajenas a todas estas acciones (Miranda, 2016). De acuerdo a esta afirmación, la Asociación Mexicana de Museos y Centros de Ciencia y Tecnología mencionaba en el año 2016 que la oferta tecnológica en museos y otros sitios de interés turístico era limitada, pero se esperaba que creciera en los próximos años.

En este contexto, y centrándonos en los dispositivos móviles, para Guerrero y Rodríguez (2016) el surgimiento de las apps, aunque sea limitado, ha impactado de forma profunda en la industria turística, de manera que el crecimiento y variedad de las mismas, ha traído nuevas alternativas de gestión turística y cultural, y desafíos para los destinos y sitios patrimoniales. Gándara (2016) concuerda con que los dispositivos móviles y las apps pueden generar nuevas oportunidades en la relación del patrimonio mexicano y sus visitantes, ya que, "la presentación digital del patrimonio no resuelve por sí misma el problema de la divulgación, pero ofrece nuevas oportunidades de comunicar los valores patrimoniales gracias a la interactividad. Con todo, para ser eficaz, Gándara (2016) cree que son necesarias nuevas tecnologías y estrategias de comunicación.

Aunque, como destacan Guerrero y Rodríguez (2016) muchos proyectos mexicanos remarcan la idea del uso de estos dispositivos con un objetivo turístico, dejando la valorización patrimonial en un segundo plano, Gándara (2017) afirma que cada vez encontramos más ejemplos de uso para el aprendizaje del patrimonio cultural, y que este se realiza principalmente a través de esas apps. Así, encontramos 8 museos mexicanos que ofertan recorridos virtuales que permiten disfrutar la arquitectura y exhibiciones desde un dispositivo móvil en cualquier sitio que cuente con una conexión a internet (DEMX, 2016). También en relación a la pujante integración de Realidad Aumentada (RA) aplicada al patrimonio (Puyuelo, Val, Merino y Felip, 2011), las apps emergen como una de las herramientas principales para su aplicación (Luna, Vicent, Cabrera y Quiñonez, 2019), habiéndose incorporado en diferentes espacios patrimoniales como el Museo Nacional de Historia y Antropología (MX guía insider, 2018), la zona arqueológica de Tulum (Instituto Nacional de Antropología e Historia, 2017) y Chichen-Itzá (Calderón, 2019), y habiéndose empezado a utilizar también en contextos de educación formal (Partida, 2016).

Pese a todo ello, en la actualidad, no existen estudios evaluativos sobre las apps de patrimonio surgidas en México, imposibilitando conocer sus características principales, modelos de comunicación y valor educativo.

\section{La evaluación de apps patrimoniales}

Como ya han concluido investigaciones previas (Crescenci-Lanna, Valente y Suárez-Gómez, 2019; Economou y Meintani, 2011), son pocos los estudios que realizan análisis exhaustivos de evaluación de apps sobre una muestra de aplicaciones digitales amplia, limitándose en muchos casos a una sola herramienta cuya implementación se prueba en un contexto específico. Esto genera un desaprovechamiento de la potencialidad educativa de este tipo de herramientas (Kukulska-Hulme, 2007). Pese a sus limitaciones, en España el estudio de apps de contenido patrimonial desde un punto de vista educativo, es un área en pleno 
desarrollo (Fontal e Ibáñez-Etxeberria, 2017; Ibáñez-Etxeberria, Fontal y Rivero, 2018), encontrando investigaciones destacables sobre evaluación de apps de educación patrimonial (Gillate, Vicent, Gómez-Redondo y Marín-Cepeda, 2017; Ibáñez-Etxeberria y Kortabitarte, 2016; Martínez-Gil, López-Benito, Asensio y Santacana, 2018). Así, desde el punto de vista de la estructuración educativa, se han publicado estudios sobre aplicaciones móviles tanto en entornos formales (Ibáñez-Etxeberria, Vicent y Asensio, 2012; Kortabitarte, Gillate, Luna e Ibáñez-Etxeberria, 2018), como informales (Asensio et al., 2013), y desde enfoques sectoriales, estudios sobre apps urbanas (Grevtsova, 2013), uso de realidad aumentada (Luna, Ibáñez-Etxeberria y Rivero, 2019; Luna, Rivero y Vicent, 2019), uso en museos de arte (López-Benito, 2013), trabajo en memoria histórica (Gillate, Luna, Castrillo e Ibáñez-Etxeberria, 2020) o inclusividad (López, Olmedo, Tadeu y Fernández, 2019). También encontramos en Italia algún trabajo experimental con RA con estudiantes (Petruco y Agostini, 2016) o en formación inicial de profesorado (Poce, Agrusti y Re, 2018).

En el caso de México, aunque se han realizado algunos estudios sobre tecnologías digitales y patrimonio (Jiménez-Badillo y Gándara, 2016) y sobre patrimonio y educación (Cantón, 2013a, 2013b; Luna et al., 2019), existe una ausencia de investigaciones de evaluación educativa de apps de patrimonio que se trataría de paliar con este estudio.

Así pues, el objetivo principal de esta investigación, se concreta en conocer el panorama de apps de patrimonio en México, analizar desde un punto de vista educativo sus características, y contextualizarlas en un contexto global más amplio derivado de la comparativa con el caso español.

Los objetivos específicos serían:

1. Inventariar y analizar las apps de Educación Patrimonial de México.

2. Comparar los resultados con las apps de educación patrimonial evaluadas en el contexto español.

\section{Método}

Esta es una investigación de tipo descriptivo basada en el estudio de programas. Para ello, se ha utilizado el método OEPE (Fontal, 2016), creado para el análisis de programas de educación patrimonial por el Observatorio de Educación Patrimonial de España, adaptado a la evaluación de apps de carácter patrimonial (Ibáñez-Etxeberria y Kortabitarte, 2016, Kortabitarte et. al., 2017). El método OEPE, es un sistema secuenciado que consta de 7 fases consecutivas y complementarias que permiten realizar la selección de la muestra en base a criterios de inclusión y exclusión (fases 1-3), un estudio de descriptivo de corte cuantitativo (fase 4), y finalmente tras la aplicación de unos estándares (fases 5 y 6), hacer una selección de casos que permite hacer un estudio de caso de corte cualitativo (fase 7). En este estudio se han completado las tres primeras fases del método OEPE (búsqueda, discriminación e inventariado), y la cuarta fase en la que se realiza el análisis de las apps a través de la adaptación de la herramienta de análisis, limitándonos a exponer el estudio cuantitativo Los datos obtenidos mediante este análisis, han sido tratados estadísticamente para obtener los datos de frecuencias y realizar el estudio descriptivo de dichos resultados.

\subsection{Muestra}

La primera fase de búsqueda e identificación del universo de apps de educación y patrimonio de México (junio-diciembre de
2018), se ha realizado en los store de Android y Apple de apps utilizando palabras clave como "patrimonio" y sus variantes, "museo", "cultura" o "tradición", completada con una búsqueda manual de elementos patrimoniales como "zona arqueológica", "cenote" o "gastronomía", los lugares más destacados (Chichen-Itzá, Tulum o Teotihuacán, ...) y ciudades y Estados de México. Este universo de apps de educación y patrimonio de México se ha completado con una búsqueda manual en otras fuentes de información como webs de equipamientos culturales, asociaciones educativas, empresas tecnológicas, de mediación educativa y cultural, equipos de investigación en educación e innovación tecnológica, hasta arrojar un listado de 85 apps.

En la segunda fase, se ha realizado la discriminación de las apps identificadas utilizando los criterios de inclusión y exclusión definidos por Ibáñez-Etxeberria y Kortabitarte (2016) adaptados al caso mexicano, de manera que se ha seleccionado la muestra definitiva de apps para dispositivos móviles referidas a patrimonio mexicano (criterios inclusivos) y que no produzca error técnico, no sea un libro digital, que no haya desaparecido de las store o que no se halle vacía de contenidos y que permita su análisis a distancia (exclusión). Esta aplicación de los filtros ha arrojado una muestra final de 45 apps susceptibles de trabajar desde el punto de vista educativo el patrimonio de México, sobre las cuales se ha procedido a ejecutar la tercera fase que conforma el inventariado de las apps aptas para el estudio. De este modo, universo de apps de educación patrimonial constaba de 85 apps, y tras la aplicación de los criterios de inclusión y exclusión la muestra final de la investigación se reduce a 45 apps (Tabla 1).

En la cuarta fase, se realiza el análisis individual de cada una de ellas aplicando la herramienta de análisis creada para el proyecto ARSMULEP, que abarca cinco dimensiones de las apps referidas a Metadatos-descriptiva, Informativo-comunicativa, Técnica, sobre Procesos de aprendizaje y Patrimonial (Kortabitarte et al., 2017). Esta herramienta consta de 103 ítems, con adaptaciones al contexto mexicano en los ítems referidos al criterio geográfico (5), al costo económico (7) y los idiomas (12). El análisis se ha realizado por dos investigadores de manera independiente (uno mexicano y uno español), y luego se han cruzado los resultados entre ellos y con otros investigadores del proyecto hasta dar con el análisis definitivo.

\section{Resultados}

Dentro de la primera dimensión referida a los metadatos, vemos que los mayores agentes impulsores de las apps en México han sido empresas privadas $(n=22 ; 44.9 \%)$, muy por encima de aquellas impulsadas por ciudadanos de manera individual $(\mathrm{m}=8 ; 16,3 \%)$ y/o universidades y grupos de investigación $(\mathrm{n}=6$; $12,2 \%$ ). Sorprende la escasa presencia de la administración pública y fundaciones, con solo 6 apps $(12,2 \%)$ y todavía menor en lo que se refiere a museos $(n=3 ; 6,1 \%)$.

Si observamos la categoría de Google Play en la que se encuentran divididas las apps de patrimonio mexicanas, un $44,4 \%$ se definen como educativas $(n=20)$, con un porcentaje parecido al de aquellas categorizadas como apps de Viajes y guías $(\mathrm{n}=18$; $40,0 \%)$. En relación a su costo, la mayor parte son gratuitas $(n=39$; $79,6 \%$ ), a excepción de 5 apps que tienen un precio entre cero y cincuenta pesos $(10,2 \%)$ y una app con un precio entre 50 y 200 pesos $(n=1 ; 2,2 \%)$. En cuanto a sistema operativo, más de la mitad están disponibles tanto para iOS como para Android ( $n=25 ; 55,6 \%$ ), mientras que sólo 6 están adaptadas a iOS (13,3\%) y 14 tan solo disponibles para Android (31,1\%). En lo que se refiere al idioma, más de la mitad están sólo en español ( $n=26 ; 57,8 \%)$, complementadas en otro $8,9 \%$ por apps bilingües en una lengua extranjera 
Tabla 1.

Muestra final de la investigación

\begin{tabular}{|c|c|c|c|c|c|}
\hline 1 & Abejas mayas & 16 & Explore Teotihuacan Español & 31 & mxplor Chichen Itza Audio Tour \\
\hline 2 & Altar de muertos & 17 & Fonoteca Itinerante & 32 & Mythology - Mayan \\
\hline 3 & Aprendiendo maya jugando & 18 & Guía Cuautla - Tourism Guide & 33 & Pistas Album \\
\hline 4 & Aves de Cozumel & 19 & Hidalgo Mágico & 34 & Puebla Tour \\
\hline 5 & Aves y Senderos de El Chico & 20 & HuitcholesMNA & 35 & Redescubre Yucatán \\
\hline 6 & Campeche en tu bolsillo & 21 & INAH Campeche & 36 & San Juan de los Lagos \\
\hline 7 & Chichen Itza Historical Guide & 22 & Jalisco Turístico & 37 & Sitio de Cuautla de 1812 \\
\hline 8 & Chichen Itza Plus- El Mentor & 23 & La máscara de Calakmul & 38 & Teotihuacan 3D \\
\hline 9 & Chichén Itzá Turismo & 24 & Leyendas de Mexico Gratis & 39 & Travel Guide YUC \\
\hline 10 & Conoce Dolores Hidalgo C.I.N. & 25 & Maya Mythology & 40 & Trivial Independencia de México \\
\hline 11 & Cosalá & 26 & Mayas. Lenguaje de la belleza & 41 & Tulum - Be Your Own Guide \\
\hline 12 & Cultura Maya Trivia & 27 & México Barroco & 43 & Turisteando Mitla \\
\hline 13 & Descubre Villa del Carbón & 28 & MexicoAlternativo & 43 & UCMUSEOS 1 \\
\hline 14 & Dioses Mayas & 29 & MonarcaMX & 44 & Vamos a aprender mixteco \\
\hline 15 & Etnoecología & 30 & Museo Paquimé & 45 & Yalam \\
\hline
\end{tabular}

$(n=4)$ en una lengua indígena $(n=4)$. De manera residual y poco contextualizada, otro $11,1 \%$ se ofrece sólo en inglés $(n=5)$

En cuanto al tipo de app en la que se categoriza (Tabla 2), encontramos un alto porcentaje de apps que sólo ofrecen información puntual ( $\mathrm{n}=22 ; 48,9 \%)$, seguidas de aquellas que sirven para realizar un autoitinerario por un museo o espacio patrimonial $(\mathrm{n}=15 ; 33,3 \%)$.

Tabla 2.

Tipo de app

\begin{tabular}{lcc}
\hline & $\mathrm{n}$ & $\%$ \\
\hline Autoitinerario & 15 & 33,3 \\
Itinerario & 2 & 4,3 \\
Juego & 6 & 13,3 \\
Información puntual & 22 & 48,9 \\
Cuento/storytelling & 0 & 0 \\
TOTAL & 45 & 100 \\
\hline
\end{tabular}

En la dimensión informativa-comunicativa, observamos que las apps analizadas apenas ofrecen espacios al usuario en los que enviar información seleccionada dentro de la aplicación, siendo solamente 4 apps las que ofrecen esta posibilidad (8,9\%), 7 apps permiten compartir información por redes sociales $(15,6 \%)$ y tan sólo 3 aplicaciones ofrecen un espacio para la creación de nuevos contenidos por parte de los usuarios $(6,7 \%)$, por lo que la oportunidad que se da a los usuarios de crear y compartir información es muy baja.

A su vez, no hemos encontrado ninguna app adaptada a diferentes niveles de usuario, y solamente $2(4,4 \%)$ están dirigidas a diferentes colectivos o tipos de usuario. Aunque la mayor parte de ellas ofrece una gramática y tipografía adecuada que permite una lectura accesible $(\mathrm{n}=26 ; 57,8 \%)$, son también escasas las apps que ofrecen lectura fácil $(n=8 ; 17,8 \%)$.

La variedad de recursos en los que se cifra la información también es limitada. Casi la mitad de las apps ofrecen un cifrado comunicativo principal basado en textos y fotos ( $n=21 ; 46,7 \%)$, encontrando en segundo lugar aquellas en las que el principal es el texto $(n=11 ; 24,4 \%)$, y en tercer lugar las que tienen mensaje verbal $(n=5 ; 11,1 \%)$.

En cuanto al discurso, un porcentaje muy alto de apps contienen un discurso informativo $(n=34 ; 75,6 \%)$ y en segundo lugar educativo $(n=9 ; 20,0 \%)$. Sin embargo, esa comunicación educativa está basada principalmente en los contenidos $(n=41 ; 91,1 \%)$ y en muy pocos casos en resultados $(n=4 ; 8,9 \%)$. Casi la totalidad de las apps muestran un tipo de comunicación informativa $(n=43$; 95,5\%).

Los resultados obtenidos en la dimensión técnica son positivos en cuanto a la usabilidad, ofreciendo gran parte de las apps un nivel de operabilidad alto $(\mathrm{n}=21 ; 46,7 \%)$ o muy alto $(\mathrm{n}=21$; $46,7 \%$ ), siendo más bajos los resultados en la calidad del entorno, encontrando pocas apps de calidad muy alta $(\mathrm{n}=13 ; 28,9 \%)$ o alta $(n=12 ; 26,7 \%)$. Cabe destacar que encontramos 11 apps de calidad muy baja $(24,4 \%)$. Tampoco encontramos datos positivos en elementos que hacen accesibles las apps, ya que solo sólo 4 (8,9\%) permiten cambiar el tamaño del texto y $6(13,3 \%)$ el de la pantalla. En este ámbito, es un poco mayor el número apps que permiten poner el texto en voiceover o lengua de signos $(\mathrm{n}=13 ; 28,9 \%)$.

La cuarta dimensión, dedicada al análisis de los procesos de aprendizaje, muestra que más de la mitad de las aplicaciones no ofrecen ningún elemento que genere engagement ( $\mathrm{n}=26 ; 57,8 \%)$ y sólo 8 apps $(17,8 \%)$ ofrecen algún tipo de interacción al usuario. La intencionalidad de estas herramientas, en coherencia con su dimensión informativa, es principalmente de difusión e informativa $(\mathrm{n}=35 ; 77,8 \%)$, con un porcentaje muy bajo de apps de intencionalidad explicativa $(n=3 ; 6,7 \%)$, interpretativa $(n=3 ; 6,7 \%)$, lúdica $(n=3 ; 6,7 \%)$ o participativa $(2,2 \%)$. 
Casi la totalidad de las apps fomenta un conocimiento memorístico $(\mathrm{n}=41 ; 91,1 \%)$, frente a 3 apps $(6,7 \%)$ explicativas y una interpretativa $(2,2 \%)$. El conocimiento que se fomenta en estas es principalmente cognitivo $(n=42 ; 93,3 \%)$ y sólo se han encontrado 2 apps $(4,4 \%)$ que fomenten el conocimiento cognitivo y afectivo y una app $(2,2 \%)$ que fomenta el aprendizaje afectivo. Si observamos el tipo de autoaprendizaje que permiten las herramientas, encontramos también resultados muy escasos, con 7 apps $(15,6 \%)$ que fomentan la reflexión, $5(11,1 \%)$ que ofrecen espacio para la creación de contenido, 4 (8,9\%) con espacios para compartir, 3 $(6,7)$ que fomentan la experimentación y simulación, y 15 apps $(33,3 \%)$ la observación.

Por último, la dimensión patrimonial ofrece resultados muy diversos. En cuanto a la perspectiva del patrimonio que ofrecen (Tabla 3), los datos más altos los encontramos en las apps que tienen una perspectiva simbólico-identitaria $(\mathrm{n}=15)$ y monumental $(\mathrm{n}=14 ; 31,1 \%)$.

Tabla 3.

Perspectiva patrimonial

\begin{tabular}{lcc}
\hline & $\mathrm{n}$ & $\%$ \\
\hline Fetichista-excepcionalista & 3 & 6,7 \\
Monumental & 14 & 31,1 \\
Estética & 3 & 6,7 \\
Temporal & 2 & 4,4 \\
Diversidad & 8 & 17,8 \\
Simbólico-identitaria & 15 & 33,3 \\
TOTAL & 45 & 100,0 \\
\hline
\end{tabular}

La finalidad de la comunicación patrimonial es principalmente academicista $(n=31 ; 68,9 \%)$, seguida de la práctica-conservacionista $(n=10 ; 22,2 \%)$. Mientras que la tipología de patrimonio a la que se refieren las apps es más variada, encontrando aquellas creadas en torno a patrimonio histórico $(n=15 ; 33,3 \%)$, etnológico $(\mathrm{n}=13 ; 28,9 \%)$, holístico $(\mathrm{n}=12 ; 26,7 \%)$, principalmente, y en menor medida apps sobre patrimonio natural $(n=3 ; 6,7 \%)$ y artístico $(n=2$; $4,4 \%$ ). Por último, los elementos constitutivos de la identidad o el patrimonio son mayoritariamente territoriales ( $n=29 ; 64,4 \%)$. El resto se refieren a una multiplicidad identitaria $(n=7 ; 15,6 \%)$, a elementos afectivos-emocionales $(n=6 ; 13,3 \%)$ y otras están relacionadas con convenciones socio-políticas ( $n=3 ; 6,7 \%)$.

\section{Discusión y conclusiones}

Uno de los primeros datos contrastables es la escasa cantidad de apps de educación patrimonial existentes en México en relación a su potencia patrimonial, ya que se han inventariado tan solo 85 apps en todo el país, de las cuales algo más de la mitad de ellas han superado los filtros establecidos para considerarlas apps educativas analizables. Este hecho constata un gran desequilibrio entre la gran cantidad de elementos patrimoniales con los que cuanta el país (Miranda, 2016), los visitantes que reciben estos espacios (Datatur, 2019), el elevado uso de dispositivos móviles e internet de la población mexicana (AMIPCI, 2018) y las herramientas digitales creadas para su conocimiento y difusión (Gándara, 2016). Estos resultados coinciden en parte con el panorama español, en el que tampoco existen muchas aplicaciones de educación patrimonial, y aquellas que son creadas con este fin tienen además una vida muy corta, desapareciendo muchas de ellas a los pocos años de su lanzamiento (Ibáñez-Etxeberria y Kortabitarte, 2016).

También es destacable que los impulsores principales de las apps mexicanas son empresas privadas, llegando a alcanzar casi la mitad de las herramientas analizadas en la muestra. No debemos olvidar la importancia de México como destino turístico (Datatur, 2019), y, por tanto, la relevancia de las empresas privadas que trabajan en torno a los espacios patrimoniales con fines lucrativos, lo que puede mandarnos un aviso sobre un posterior desarrollo que puede ir in crescendo.

Por el contrario, aun siendo los custodios de los elementos patrimoniales del país (Miranda, 2016), la administración pública y los museos ocupan los últimos puestos, lo que confirma lo descrito por Gándara (2016), quien afirma que el trabajo de INAH, por ejemplo, se ha dirigido a la investigación o la restauración de los innumerables bienes culturales del país, dejando a un lado la socialización de dichos elementos, y reafirmando las conclusiones de Cantón (2013b), que destaca las escasas investigaciones en torno a la educación patrimonial. Esta realidad se encuentra en contraposición con el contexto español, donde según los datos obtenidos por Ibáñez-Etxeberria y Kortabitarte (2016) y Gillate et al. (2017), la administración pública y los museos son los principales impulsores de las apps, encontrándose los museos en los últimos puestos de agentes desarrolladores y en los que la investigación sobre educación patrimonial y nuevas tecnologías se encuentra en pleno auge (Fontal e Ibáñez-Etxeberria, 2017; Ibáñez-Etxeberria et al., 2018).

Esta situación provoca, además, que las empresas dedicadas al turismo creen apps centradas en una difusión de la información muy superficial, normalmente sobre los destinos turísticos principales y en los que se mezclan contenidos patrimoniales con comerciales. Estas herramientas, en muchas ocasiones, repiten esquemas de folletos o carteles turísticos ya obsoletos pero adaptados a dispositivos móviles (Grevtsova, 2013), sin poder considerarse herramientas educativas, y donde los diseños didácticos

apenas tienen cabida, tal y como sucede en el caso de las aplicaciones españolas (Gillate et al., 2017; Martínez-Gil et al., 2018). Este hecho se contrapone con la categoría en la que se engloban las apps analizadas, las cuales se autocalifican en primer lugar en la categoría de educación de Google Play tanto en las apps analizadas en el proyecto EPAMEX como en el último estudio realizado sobre aplicaciones españolas (Gillate et al., 2017), lo cual nos permite intuir una visión condescendiente y desinformada sobre lo que supone la educación patrimonial.

Esto ayuda a comprender que casi la mitad de las apps analizadas no muestren elementos de engagement, y que apenas incluyan juegos o storytellings que puedan conectar mejor con el usuario. La mayoría de apps son itinerarios u ofrecen información puntual, tal y como sucede en España, por lo que el usuario se convierte en un agente pasivo que apenas participa en la creación de contenidos o toma de decisiones. Del mismo modo, no se incluyen actividades que fomenten el autoaprendizaje. Por consiguiente, se obvia la idea de que los medios -en este caso tecnológicos- no son solo la finalidad, sino que debe dársele importancia al uso que de ellos se hace (Gándara, 2017). Así, las apps mexicanas analizadas se presentan como una herramienta de la cual no se aprovechan sus funcionalidades educativas, sino que se convierten en meros productos que deben ofrecerse al público por el hecho de ser tecnologías novedosas, sin importar los contenidos y procesos que en ellas deben desarrollarse. Del mismo modo, el usuario anónimo al que se le ofrecen contenidos sin adaptación didáctica, no encuentra en estas apps un medio accesible ni adaptado a las diferentes tipologías de usuario que pueden hacer uso de ellas (Gillate et al., 2017; Martínez-Gil et al., 2018). 
Por último, encontramos mayores diferencias entre las apps mexicanas analizadas y aquellos datos obtenidos en España en lo referido a la perspectiva patrimonial que ofrecen las apps. Los resultados variados están condicionados por la visión diversa en torno al patrimonio en los diferentes países, ya que tal y como hemos mencionado previamente las investigaciones en torno a la educación y el patrimonio, han obtenido gran relevancia en España durante los últimos años (Fontal, 2016; Ibáñez-Etxeberria et al., 2018), a diferencia de lo que sucede en México (Cantón, 2013a; 2013b).

La perspectiva simbólico-identitaria alcanza los valores más altos, así como los monumentales, relacionado con la gran cantidad de sitios arqueológicos que se encuentran en el país, así como la relevancia del patrimonio inmaterial inventariado en las listas de la UNESCO, muchas de ellas relacionadas con los grupos indígenas (UNESCO, 2017). Estos resultados difieren de la concepción del patrimonio observada en las apps españolas, donde la visión estética y monumental son aquellas que alcanzan mayor representación (Ibáñez-Etxeberria y Kortabitarte, 2016). Sin embargo, si encontramos similitudes en la tipología principal sobre la que se han creado las apps, siendo en ambos casos el patrimonio histórico al que más se hace referencia, siendo también la visión holística del patrimonio un aspecto destacable.

\section{Referencias bibliográficas}

AMIPCI (2018). $14^{\circ}$ estudio sobre los hábitos de los usuarios de internet en México. México: Asociación Mexicana de Internet. Recuperado de: https://www.asociaciondeinternet.mx/ es / component/remository/Habitos-de-Internet/14-Estudio-sobre-los-Habitos-de-los-usuarios-de-Internet-en-Mexico-2018/lang,es-es/?Itemid=

Asensio, M., Castro, Y., Asenjo, E., Pol, E., Rodríguez, J.A., Paredes, P., Cabrera, A., Rodríguez, I., y Villar, C. (2013). Cómo aprender disfrutando de la 'Cocina Valeniana': un modelo de evaluación para el diseño de dispositivos de realidad aumentada. En A. Cabrera, A. Rodríguez y Villar, C. (Eds.). La cocina valenciana del Museo Nacional de Artes Decorativas. Una relectura a través de la tecnología de Realidad Aumentada (pp. 153-187). Madrid: Secretaria de Estado de Cultura. https:// www.academia.edu/33972370/C\%C3\%B3mo aprender disfrutando de la Cocina Valenciana un modelo de evaluaci $\%$ C $3 \%$ B3n para el dise $\%$ C $3 \%$ B10 de dispositivos de Realidad Aumentada

Calderon, N. (21 de agosto de 2019). Propone INAH poner códigos QR a los vestigios mayas. Novedades de Yucatán. Recuperado de: https://sipse.com/novedades-yucatan/zonas-arqueologicas-yucatan-inah-codigos-qr-341827.html

Cantón, V. (2013a). Desarrollo de la educación patrimonial en México. Una propuesta de periodización. Correo del maestro, 209, 30-46.

Cantón, V. (2013b). La educación patrimonial: Educar con y para el patrimonio. Primera parte. Correo del maestro, 206, 42-51.

Crescenzi-Lanna, L., Valente, R. y Suárez-Gómez, R. (2019). Aplicaciones educativas seguras e inclusivas: La protección digital desde una perspectiva ética y crítica. Comunicar, 61, 93-102. https://www.revistacomunicar.com/verpdf. php?numero $=61 \&$ articulo $=61-2019-08$

Datatur (2019). Resultados de la Actividad Turística Julio, 2019. Subsecretaría de Planeación y Política Turística. Recuperado de: http://www.datatur.sectur.gob.mx/SitePages/versionesRAT.aspx

DEMX (8 de junio de 2016). 8 museos de México que tienen re- corridos virtuales para ti. Recuperado de: https://masdemx. com/2016/06/8-museos-de-mexico-que-tienen-paseos-virtuales-para-ti/

Economou, M., y Meintani, E. (2011). Promising beginnings? Evaluating museum mobile phone APPs. In Re-thinking technology in museums 2011. Emerging Experiences, Proceedings of the International Conference, University of Limerick, Ireland, 26-27 May 2011; Luigina Ciolfi, Katherine Scott, Sara Barbieri, Eds.; University of Limerick: Limerick, Ireland 2011; 87-101. Recuperado de: www.idc.ul.ie/techmuseums11/paper/paper8.pdf/

Fontal, O. (2016). The Spanish Heritage Education Observatory. Cultura y Educación, 28(1). https://www.tandfonline.com/ doi/abs/10.1080/11356405.2015.1110374

Fontal, O. e Ibañez-Etxeberria, A. (2017). La investigación en Educación Patrimonial. Evolución y estado1 actual a través del análisis de indicadores de alto impacto. Revista de educación, 375, 184-214 https://sede.educacion.gob.es/publiventa/descarga.action?f codigo agc $=18213$

Gándara, M. (2016). ¿Difundir o divulgar? He ahí el dilema. En D. Jiménez-Badillo y M. Gándara Vázquez (Eds.). El patrimonio cultural y las tecnologías digitales: experiencias recientes desde México (pp. 60-73). México: Instituto Nacional de Antropología e Historia-CONACYT. https://www.academia. edu/32228784/ Difundir o divulgar he ah\%C3\%AD el dilema

Gándara, M. (2017). Nuevas tecnologías y estrategias de comunicación para la divulgación del patrimonio cultural. ANTROPOLOGÍA. Revista interdisciplinaria del INAH, 1(1), 135-153. https://www.academia.edu/34088572/Nuevas tecnologi as y estrategias de comunicacio $n$ para la divulgaci $\%$ C3\%B3n del patrimonio cultural

Gillate, I.; Luna, U.; Castrillo, J. e Ibáñez-Etxeberria, A. (2020) Historical Memory in Heritage Education Apps: A resource to work on social and civic competence. En Handbook of Research on Citizenship and Heritage Education (285-310). IGI Global Publisher

Gillate, I., Vicent, N., Gómez- Redondo, C., y Marín- Cepeda, S. (2017). Características y dimensión educativa en apps de educación patrimonial. Análisis a partir del método OEPE. Revista de Estudios Pedagógicos, 43(4), 115-136. Recuperado de: http://revistas.uach.cl/index.php/estped/article/ view $/ 1793$

Gobierno de México (2018). Comunicado de prensa. Encuesta Nacional sobre Disponibilidad y Uso de Tecnologías de la Información en los hogares. Recuperado de: http://www.beta. inegi.org. $\mathrm{mx} /$ contenidos/saladeprensa/boletines/2018/ OtrTemEcon/ENDUTIH2018 02.pdf

Grevtsova, I. (2013). El patrimonio urbano al alcance de la mano: arquitectura, urbanismo y apps. HerEMus, 13, 33-46 https:// raco.cat/index.php/Hermus/article/view/313392/403511

Guerrero Rodríguez, R. y Rodríguez Zavala, D. (2016). Desarrollo de tecnología móvil en sitios de interés patrimonial proyecto: Teatro Juárez en Guanajuato. En D. Jiménez-Badillo y M. Gándara Vázquez (Eds.). El patrimonio cultural y las tecnologías digitales: experiencias recientes desde México (pp. 270-285). México: Instituto Nacional de Antropología e Historia-CONACYT.

Ibáñez-Etxeberria, A., Fontal, O. y Rivero, P. (2018). Educación patrimonial y TIC en España: marco normativo, variables estructurantes y programas referentes. Arbor, 194(788) http://arbor.revistas.csic.es/index.php/arbor/article/ view $/ 2261 / 3184$

Ibáñez-Etxeberria, A. y Kortabitarte, A. (2016). Apps, Redes Socia- 
les y dispositivos móviles en Educación Patrimonial. Recuperado de http://www.mecd.gob.es/planes-nacionales/dam/ jer:4cc8d5a2-3084-409e-a134-b46b62f7126e/apps-y-socialmedia-en-educacionpatrimonial.pdf

Ibáñez-Etxeberria, A.; Vicent, N. y Asensio, M. (2012). Aprendizaje informal, patrimonio y dispositivos móviles. Evaluación de una experiencia en educación secundaria. Didáctica de las Ciencias Experimentales y Sociales. 26, 3-18. https://ojs.uv.es/ index.php/dces/article/view/1937/1441

Instituto Nacional de Antropología e Historia (2 de abril de 2017). Preparan aplicación de realidad aumentada para la Zona Arqueológica de Tulum. Recuperado de: https://inah.gob.mx/ boletines /6029-preparan-aplicacion-de-realidad-aumentada-para-la-zona-arqueologica-de-tulum

Jiménez-Badillo, D. (2016). La Redtdpc y la difusión del patrimonio cultural en el siglo XXI. En D. Jiménez-Badillo y $\mathrm{M}$. Gándara Vázquez (Eds.). El patrimonio cultural y las tecnologías digitales: experiencias recientes desde México (pp. 42-59). México: Instituto Nacional de Antropología e Historia-CONACYT.

Jiménez-Badillo, D., y M. Gándara Vázquez, M. (Eds.) (2016). El patrimonio cultural y las tecnologías digitales: experiencias recientes desde México. México: Instituto Nacional de Antropología e Historia-CONACYT.

Kortabitarte, A., Gillate, I., Luna, U., e Ibáñez-Etxeberria, A. (2018). Las aplicaciones móviles como recursos de apoyo en el aula de Ciencias Sociales: Estudio exploratorio con el app "Architecture gothique/romane" en Educación Secundaria. ENSAYOS. Revista de la Facultad de Educación de Albacete, 33囚1, 65-79. https://revista.uclm.es/index.php/ensayos/article/ view $/ 1743 / \mathrm{pdf}$

Kortabitarte, A., Ibañez-Exeberria, A., Luna, U., Vicent, N., Gillate, I., Molero, B. y Kintana, J. (2017). Dimensiones para la evaluación de aprendizajes en APPs sobre patrimonio. Pulso: Revista de educación, 40, 17-33. Recuperado de: https:// revistas. cardenalcisneros.es/index.php/PULSO/article/view/252

Kukulska-Hulme, A. (2007). Mobile Usability in Educational Contexts: What have we learnt? The International Review of Research in Open and Distributed Learning, 8(2). Recuperado de: http://www.irrodl.org/index.php/irrodl/rt/printerFriendly $/ 356 / 879$

López Benito, V. (2013). ¿Educar en el arte mediante apps? Los museos de arte y el uso de aplicaciones móviles en el contexto español. HerEMus: heritage $\mathcal{E}$ museography, 13, 6974. https://www.raco.cat/index.php/Hermus/article/ view $/ 313408 / 403527$

López Delgado, A., Olmedo, E., Tadeu, P. y Fernández Batanero, J. M. (2019). Propuesta de las condiciones de las Aplicaciones móviles, para la construcción de un Entorno de Accesibilidad Personal para usuarios con discapacidad visual en las Smart Cities. Aula Abierta, 48(2), 193-202. DOI: https://doi. org/10.17811/rifie.48.2.2019. Recuperado de: https://www. unioviedo.es/reunido/index.php/AA/issue/view/1124/ showToc

Luna, U., Ibáñez-Etxeberria, A., Rivero, P. (2019). El patrimonio aumentado. 8 apps de Realidad Aumentada para la enseñanza-aprendizaje del patrimonio. Revista Interuniversitaria de Formación del Profesorado, 94(33.1), 43-62. Recuperado de: https://recyt.fecyt.es/index.php/RIFOP/article/view/72088

Luna, U., Rivero, P. y Vicent, N. (2019). Augmented Reality in Heritage Apps: Current Trends in Europe. Applied Sciences, 9(2756), 1-15. doi:10.3390/app9132756

Luna, U., Vicent, N., Reyes Cabrera, W. y Quiñonez Pech, S.H. (2019). Patrimonio, currículum y formación del profesorado de Educación Primaria en México. Revista Electrónica Interu- niversitaria de Formación del Profesorado, 22(1),83-102. Recuperado de: https://revistas.um.es/reifop/article/view/358761

Martínez-Gil, T., López-Benito, V., Asensio, M., y Santacana, J. (2018). ¿Entretener o aprender? La evaluación de las tecnologías móviles en la Didáctica del Patrimonio. ENSAYOS, Revista de la Facultad de Educación de Albacete, 33(1). Recuperado de: http://www.revista.uclm.es/index.php/ensayos

Miranda, E. (2016). La Red Temática: orígenes y desarrollo. En D. Jiménez-Badillo y M. Gándara Vázquez (Eds.). El patrimonio cultural y las tecnologías digitales: experiencias recientes desde México (pp. 34-41). México: Instituto Nacional de Antropología e Historia-CONACYT.

MX guía insider (2018). El museo nacional de antropología es el primer museo en usar realidad aumentada. Recuperado de: https://mxcity.mx/2018/05/el-museo-nacional-de-antropologia-es-el-primer-museo-en-usar-una-app-de-realidad-aumentada/

Partida, J.M. (2016). Preservación del patrimonio cultural de México. Algunos factores psicopedagógicos en la incorporación de la realidad aumentada en la educación preescolar. En D. Jiménez-Badillo y M. Gándara Vázquez (Eds.). El patrimonio cultural y las tecnologías digitales: experiencias recientes desde México (pp. 106-119). México: Instituto Nacional de Antropología e Historia-CONACYT.

Petrucco, C. Y Agostini, D. (2016) Teaching our cultural heritage using mobile augmented reality, Journal of e-Learning and Knowledge Society (12)3, 115-128. https://www.learntechlib. org/p/173477/

Poce, a., Agrusti, F. y Re, M.R. (2018) Heritage education and initial teacher training: An international experience, Journal of e-Learning and Knowledge Society (14)2, 127-143. https:// www.learntechlib.org/p/184460/

Puyuelo, M., Val, M., Merino, L., y Felip, F. (2011). Representaciones virtuales y otros recursos técnicos en la accesibilidad al patrimonio cultural. Revista de expresión gráfica arquitectónica, 16(17), 164-173. https://polipapers.upv.es/index.php/ EGA/article/view/919/988

RedTDPC (2016). Red Temática Tecnologías Digitales para la Difusión del Patrimonio Cultural [página web]. Recuperado de: http://newsnet.conacytprensa.mx/index.php/documentos/10041-tecnologi-as-digitales-para-la-difusio-n-del-patrimonio-cultural

Sectur, Secretaría de Turismo (2011). Programa ruta de los Dioses. Recuperado de: https://web.archive.org/ web/20140820070850/http://www.sectur.gob.mx/es/sectur/sect Programa Ruta de los Dioses

Sectur, Secretaría de Turismo (2017). Compendio estadístico del turismo en México, 2017. Subsecretaría de Planeación y Política Turística. Dirección General de Integración de Información Sectorial. Recuperado de: https://www.datatur.sectur. gob.mx/SitePages/CompendioEstadistico.aspx

UNESCO (2017). UNESCO México [página web]. Recuperado de: http://www.unesco.org/new/es/mexico/work-areas/ culture/world-heritage/ 